\title{
Another dimension to the understanding of local therapy for metastatic prostate cancer
}

\author{
Michael Leveridge, MD, FRCSC; Robert J. Hamilton, MD, FRCSC ${ }^{2}$ \\ 'Departments of Urology and Oncology, Queen's University, Kingston, ON, Canada; '2Division of Urology, University of Toronto, Toronto, ON, Canada
}

Cite as: Can Urol Assoc J 2019;13(6):182-3. hitp://dx.doi.org/10.5489/cuaj.6049

See related article on page 175

W e find ourselves at last with a nascent understanding that local therapy (LT) for patients with metastatic prostate cancer may confer a survival benefit. Retrospective and administrative data have given us due suspicion, but the results of the 819-patient, low-volume subset of the sprawling STAMPEDE trial, with a hazard ratio (HR) for death of 0.68 (95\% confidence interval [Cl] 0.52 $0.90)$ favouring radiation therapy (RT), are the first prospective data showing such a benefit. ${ }^{1}$ Of note of course, is that its immediate predecessor, HORRAD (HR 0.90 [0.70-1.14]) and the undifferentiated cohort of STAMPEDE patients (HR $0.92[0.80-1.06]$ ) did not find a benefit to RT in metastatic patients, although the underpowered HORRAD cohort, with $<5$ metastases, had an identical HR of 0.68 to STAMPEDE's low-volume group. ${ }^{1,2}$ More patients are currently being randomized and followed in other trials, including radical prostatectomy (RP) in addition to RT, that should bring us closer to the correct answer.

Into this mix arrives the present study in this issue of CUAJ on the impact of race on patients receiving LT for their metastatic disease, an area of significant interest in which the above-mentioned trials shed no light. ${ }^{3}$ This highly regarded group of authors interrogated Surveillance, Epidemiology, and End Results (SEER) data and identified 529 men with both LT and metastatic prostate cancer, and determined that among those managed with RP, African American (AA) patients lived less than half as long (46 months vs. 108 months; HR for overall mortality 0.60). No such difference was found overall $(p=0.07)$, driven by the lack of difference in the RT group.

Over-SEERing quality meat risks drying it out, and indeed it is almost a journal club sport to dismantle registry-based studies on account of the risks of selection bias and the vagaries of coding. Before scratching that itch, it is important to recognize that the possibility of confounding does not define the existence of confounding. All of these patients received $L T$, and so a major source of confounding (the decision regarding use of $L T$ in the first place) has been obviated. In the case of the studied outcome, understanding that there are likely disease biology and care disparities between ethnicities should remain front of mind from trial design through health policy decision-making. The worse survival in the RP cohort among AA patients is stark and likely not offset by the differences between them and the Caucasian cohort, specifically that higher prostate-specific antigen (PSA) (18.2 vs. 13.4) and larger burden of visceral metastatic disease (M1c $26.4 \%$ vs. $20.3 \%$ ) suggest a more advanced or aggressive disease phenotype in the AA group, or identification of the disease at a later stage in these patients. These data are congruent with robust prior data of more adverse prostate cancer in AA men. ${ }^{4}$

In addition to the study of racial disparity, this is a cohort study on the impact of LT in metastatic patients. In the emerging era of prospective data in this space, registry studies will carry less weight in the canon, especially with concerns about SEER's granularity in defining treatments and initial disease burden. The current study is not portrayed, nor obviously intended, as a study of the efficacy of LT in metastatic prostate cancer (there is no control arm, for example), but as a data source in this area, it merits some scrutiny. The RT patients here were solely managed with brachytherapy based on the inability of the dataset coding to parse metastasis-directed from local external beam treatment (EBRT). We thus cannot compare to HORRAD nor STAMPEDE, which both involved only EBRT.

These patients fared better than their randomized peers; median survival of 82 months and 57 months in Caucasian and AA patients, respectively, are higher than the treated cohorts in HORRAD and STAMPEDE (45 months and 48 months, respectively). They also survived dramatically longer than the placebo-treated patients in the CHAARTED and LATITUDE trials of newly diagnosed metastatic patients (44 months and 34.7 months, respectively), who might best represent the expected outcomes of such patients in the 20042014 era of the study. ${ }^{5,6}$ They are likely a highly selected 
group of "favourable risk" patients. The high proportion of visceral disease $(21.7 \%$ overall) is also in contradistinction to the STAMPEDE cohort, among whom $6.5 \%$ had lung or liver metastases.

Since indications and disease burden are unknown in the current dataset, we cannot place them as high- or low-volume, as we currently define metastatic burden in CHAARTED and LATITUDE. Booth and colleagues raised interesting points in reviewing a similar study of bladder cancer patients treated with LT. ${ }^{7}$ They note that LT in a low-literature precedent setting is likely to be performed in patients with good performance status, low disease burden, and high risk of significant local complication from their cancer. They also highlight the chance that a meaningful number of patients may have been presumed to have localized disease at treatment, with metastases discovered early enough in the post-treatment period that coding into administrative data would report them as metastatic. These may result in a study population enriched for low-volume disease and improved outcome. The curiously high rate of visceral metastases in this cohort might speak to a different disease phenotype or might conflate with coding (or even diagnostic) issues around the true state of the disease.

As the appropriate evidence-based application of LT to metastatic prostate cancer patients crystallizes in upcoming years, the applicability of the results and recommendations to the broader demographics of the real world, outside the classically under-representative randomized trial population, will remain critical. The authors have added freight to the evidence supporting care disparities affecting minority popu- lations and an additional voice to the call for optimization and appropriate distribution of care.

Competing interests: Dr. Leveridge has been an advisory board member for Astellas. Dr. Hamilton reports no competing personal or financial interests related to this work.

\section{References}

1. Parker $C($, James ND, Brawley $C D$, et al. Radiotherapy to the primary tumour for newly diagnosed, metastatic prostate cancer (STAMPEDE): A randomized controlled phase 3 trial. Lancet 2018;392:2353-66. https://doi.org/10.1016/S0140-6736(18)32486-3

2. Boevé L, Hulshof MN, Vis A, et al. Effect on survival of androgen deprivation therapy alone compared to androgen-deprivation therapy combined with concurrent radiation therapy to the prostate in patients with primary bone metastatic prostate cancer in a prospective randomized clinical trial: Data from the HORRAD trial. Eur Urol 2019;75:410-8. https://doi.org/10.1016/i.eururo.2018.09.008

3. Mazzone E, Bandini $M$, Preisser $F$, et al. The effect of race on survival after local therapy in metastatic prostate cancer patients. Can Urol Assoc J October 15; Epub ahead of print. http://dx.doi.org/10.5489/ cuaj.5399

4. Chornokur G, Dalton K, Borysova $M$, et al. Disparities at presentation, diagnosis, treatment, and survival in African American men affected by prostate cancer. Prostate 2011;71:985-97. https://doi.org/10.1002/pros.21314

5. Sweeney CJ, Yu-Hui C, Carducci $M$, et al. Chemohormonal therapy in metastatic hormone-sensitive prostate cancer. New Engl J Med 2015;373:737-46. https://doi.org/10.1056/NEJMoa1503747

6. Fizazi K, Tran N, Fein L, et al. Abiraterone plus prednisone in metastatic, castration-sensitive prostate cancer. New Engl J Med 2017;377:352-60. https://doi.org/10.1056/NEJMoal704174

7. Booth CM, Karim S, Peng Y, et al. Radical treatment of the primary tumour in metastatic bladder cancer: Potentially dangerous findings from observational data. J Clin Oncol 2018;36:533-5. https://doi.org/10.1200/JC0.2017.76.1759

Correspondence: Dr. Michael Leveridge, Departments of Urology and Oncology, Queen's University, Kingston, ON, Canada; michael.leveridge@kingstonhsc.ca 